\title{
Isotopic dependence of predissociation linewidths in the Schumann- Runge bands of oxygen
}

\author{
A. S-C. Cheung and D. K-W. Mok \\ Department of Chemistry, The University of Hong Kong, Hong Kong \\ K. Yoshino, W. H. Parkinson, M. J. Jamieson, ${ }^{\text {a) }}$ and A. Dalgarno \\ Harvard-Smithsonian Center for Astrophysics, Cambridge, Massachusetts 02138 \\ M. S. Child \\ Department of Theoretical Chemistry, University of Oxford, Oxford, United Kingdom
}

(Received 16 March 1995; accepted 3 May 1995)

Recent work on predissociation in the Schumann-Runge $\left(B^{3} \Sigma_{u}^{-}-X^{3} \Sigma_{g}^{-}\right)$system of oxygen ${ }^{1-4}$ encourages a study of the isotopic dependence of the linewidths. It has been shown that the predissociation is dominated by the spin-orbit coupling to the repulsive ${ }^{5} \Pi_{u}$ state with smaller contributions from couplings to the ${ }^{3} \Pi_{u},{ }^{1} \Pi_{u}$ and ${ }^{3} \Sigma_{u}^{+}$states. ${ }^{5}$ Additionally the orbit-rotation interaction between the $B{ }^{3} \Sigma_{u}^{-}$ and ${ }^{3} \Pi_{u}$ states has been demonstrated to be important for lines emanating from high rotational states. ${ }^{6}$ In this Note we describe the isotopic dependence of the linewidths.

In Mulliken's classification of predissociation ${ }^{7}$ the crossing of the potential energy curves of the ${ }^{3} \Pi_{u}$ and $B{ }^{3} \Sigma_{u}^{-}$ states is of type $C^{-}$(where the predissociating state crosses the repulsive branch of the bound state) and crossings of the ${ }^{5} \Pi_{u},{ }^{1} \Pi_{u}$ and ${ }^{3} \Sigma_{u}^{+}$states are of type $C^{+}$(where the predissociating states cross the attractive branch). Within the semiclassical approximation the width $\Gamma_{v}$, caused by one of the predissociating states, of the vibrational level with quantum number $v$ and energy $E_{v}$ (measured from the bottom of the well) satisfies ${ }^{8-10}$

$$
\frac{\Gamma_{v}}{\hbar \omega_{v}}=\pi C\left(\frac{3}{2} \phi_{v}\right)^{1 / 3}\left(E_{v}-E_{x}\right)^{-1 / 2} \mathrm{Ai}^{2}\left[-\left(\frac{3}{2} \phi_{v}\right)^{2 / 3}\right],
$$

where $\hbar \omega_{v}\left(=d E_{v} / d v\right)$ and $C$ are given in Ref. $9, \phi_{v}$ is a phase (given in Ref. 9 for a $C^{+}$crossing and in Ref. 10 for a $\mathrm{C}^{-}$crossing), $E_{x}$ is the energy at the crossing point and $\mathrm{Ai}$ denotes the Airy function. We have previously described the calculation of the predissociation linewidths for oxygen based on a least squares fit to experimental data. ${ }^{4}$ Letting $\mu$ denote the ratio of the reduced mass for any one of the three oxygen isotopomers ${ }^{16} \mathrm{O}_{2},{ }^{16} \mathrm{O}^{18} \mathrm{O}$ and ${ }^{18} \mathrm{O}_{2}$ to that of the reference isotopomer ${ }^{16} \mathrm{O}_{2}$ we see, from Ref. 9, that the phases $\phi_{v}$ and the quantity $C$ scale as $\mu^{1 / 2}$. Hence the characteristic energy $E^{*}$ in the (linear) approximation, ${ }^{9}$

$$
\left(\frac{3}{2} \phi_{v}\right)^{2 / 3} \approx \frac{\left(E_{v}-E_{x}\right)}{E^{*}}
$$

scales as $\mu^{-1 / 3}$. Within this approximation we can determine the dependence of the width on the energy $E_{v}$. This dependence may be expressed as

$$
\frac{\Gamma_{v}}{\hbar \omega_{v}}=K \mu^{2 / 3} \mathrm{Ai}^{2}\left[-\mu^{1 / 3} \frac{\left(E_{v}-E_{x}\right)}{\tilde{E}^{*}}\right],
$$

where $K$ is mass independent and $\tilde{E}^{*}$ is the value of $E^{*}$ for the reference isotopomer ${ }^{16} \mathrm{O}_{2}$. Expression (1) goes over smoothly to sinusoidal variation for $E \gg E_{x} .{ }^{9}$ Within approximation (2) this becomes

$$
\frac{\Gamma_{v}}{\hbar \omega_{v}}=\frac{K\left(\tilde{E}^{*}\right)^{1 / 2}}{\pi} \mu^{1 / 2}\left(E_{v}-E_{x}\right)^{-1 / 2} \sin ^{2}\left(\mu^{1 / 2} \tilde{\phi}_{v}+\frac{\pi}{4}\right),
$$

where $\tilde{\phi}_{v}$ is the phase for the reference isotopomer ${ }^{16} \mathrm{O}_{2}$.

Equations (3) and (4) show that the widths depend in a complicated manner on the reduced mass and do not have the simple dependence we implied. ${ }^{4}$ Figure 2 of Ref. 4 could be viewed to imply that the widths can be fitted to a universal curve for all isotopomers. However this conclusion is ambiguous because the points are fortuitously distributed so as to obscure the mass dependence. Furthermore Fig. 2 of Ref. 4 shows the total widths; more precise information is obtained from the individual linewidths. We illustrate this briefly with a sample of calculated widths for two predissociating states: the ${ }^{3} \Pi_{u}$ of type $C^{-}$where the Airy fit (3) is appropriate and the ${ }^{5} \Pi_{u}$ where the sine fit (4) is appropriate.

Figure 1 shows the ${ }^{3} \Pi_{u}$ widths for each isotopomer. It is clear that all three sets of widths do not fit a universal curve. This was obscured in the plot in Ref. 4 of the total widths. The energies were calculated from equation (5) below.

Our ${ }^{5} \Pi_{u}$ widths are in the sinusoidal region where equation (4) is appropriate. Because of the disposition of points it is not so obvious from a direct fit that each isotopomer fits a unique curve. However equation (4) clearly shows a mass dependence. To show that the linear semiclassical approximation, which leads to equation (4), is appropriate for our ${ }^{5} \Pi_{u}$ calculations we have calculated the corresponding phases and we show, in Fig. 2, that the quantity $\left(\frac{3}{2} \phi_{v}\right)^{2 / 3} \mu^{-1 / 3}$ plotted as a function of energy $E_{v}$, has the linear form of equation (2). The energies were calculated from Ref. 11

$$
\begin{aligned}
E_{v}= & \left(v+\frac{1}{2}\right) \omega_{e} \mu^{-1 / 2}-\left(v+\frac{1}{2}\right)^{2} \omega_{e} x_{e} \mu^{-1} \\
& +\left(v+\frac{1}{2}\right)^{3} \omega_{e} y_{e} \mu^{-3 / 2},
\end{aligned}
$$

where $\omega_{e}$ was taken as $709.31 \mathrm{~cm}^{-1}, \omega_{e} x_{e}$ as $10.65 \mathrm{~cm}^{-1}$ and $\omega_{e} y_{e}$ as $-0.139 \mathrm{~cm}^{-1}$. The dependence of $E_{v}$ on $\mu$, being dominated by the first term on the right hand side of equation (5), can be largely removed by simple scaling. Equation (5) also provides $\hbar \omega_{v}=d E_{v} / d v$. The values of 


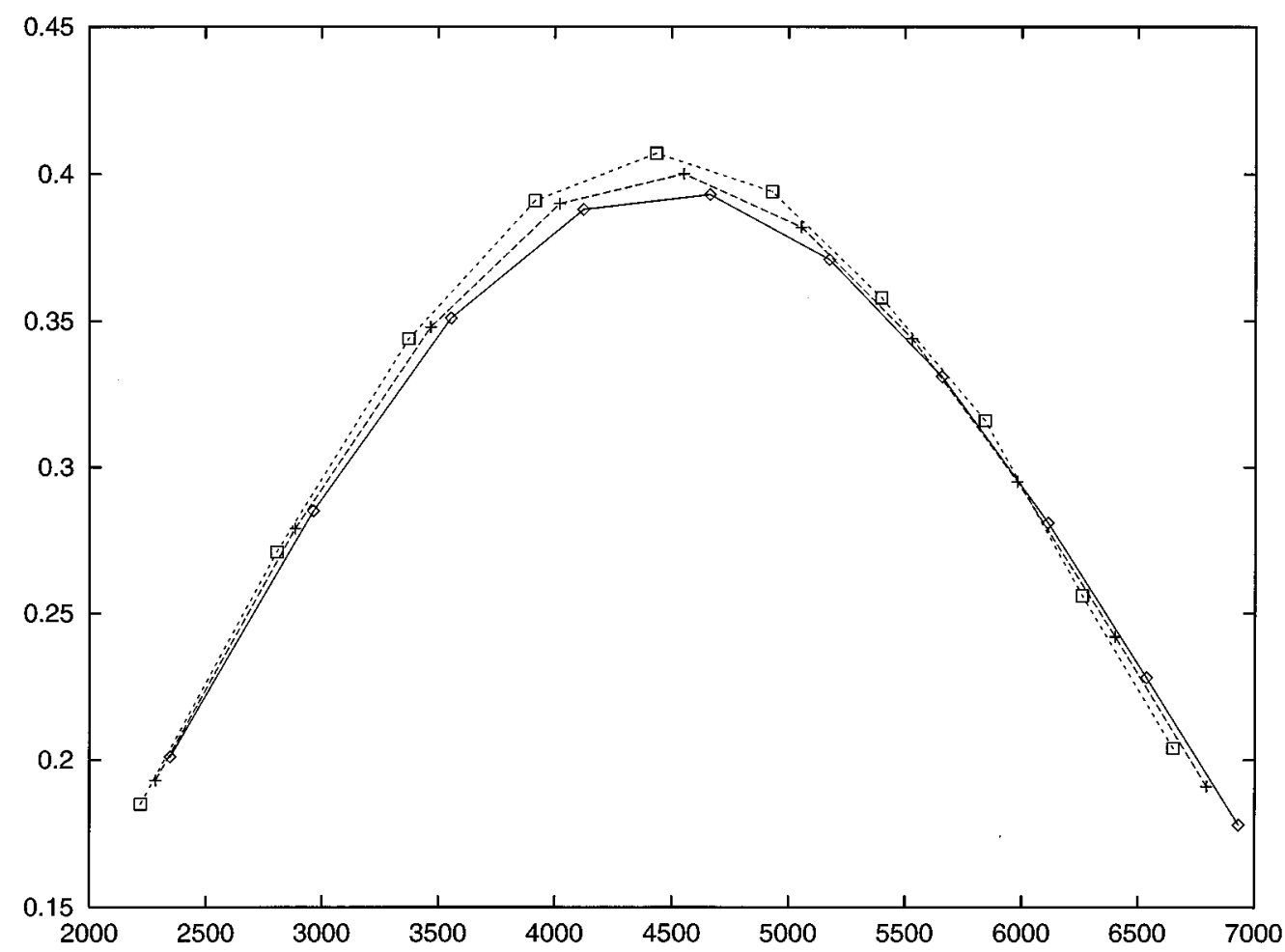

FIG. 1. Width $\left(\mathrm{cm}^{-1}\right)$ vs energy $\left(\mathrm{cm}^{-1}\right)$ in predissociation by the ${ }^{3} \Pi_{u}$ state.

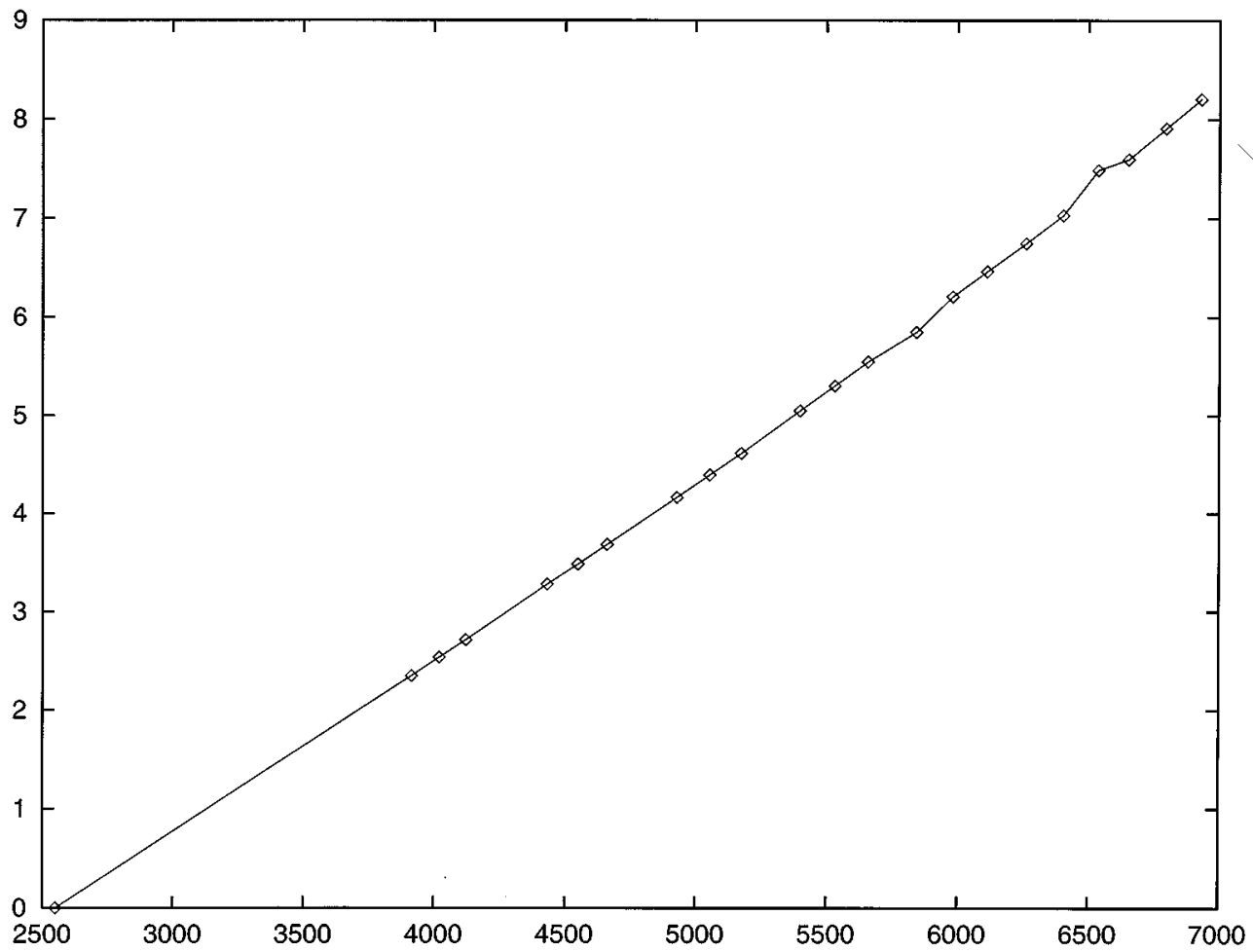

FIG. 2. $\left(\frac{3}{2} \phi_{v}\right)^{2 / 3} \mu^{-1 / 3}$ vs energy $\left(\mathrm{cm}^{-1}\right)$ in predissociation by the ${ }^{5} \Pi_{u}$ state. 
$\tilde{E}^{*}=573 \mathrm{~cm}^{-1}, E_{x}=2550 \mathrm{~cm}^{-1}$ and $K=4.481$ were calculated by the method described in Ref. 10. The graph of the dimensionless quantities $\left(\frac{3}{2} \phi_{v}\right)^{2 / 3} \mu^{-1 / 3}$ and the intercept $\left(E_{x}, 0\right)$ vs energies is expected to be a line with slope $1 / \tilde{E}^{*}$. Figure 2 demonstrates this; from the slope we find the value of $\tilde{E}^{*}$ to be $557 \mathrm{~cm}^{-1}$ compared with $573 \mathrm{~cm}^{-1}$ given above. The $v=11$ vibrational level of ${ }^{16} \mathrm{O}_{2}$ and the $v=10$ vibrational level of ${ }^{18} \mathrm{O}_{2}$ are off line. This occurs because in each of these cases the phase is $\pi / 4$ greater than a multiple of $\pi$ causing its evaluation from the width to be ill conditioned.

In conclusion we see that in semiclassical theory, in contrast to the vibrational energy levels, the dependence of the predissociation widths on the reduced mass cannot be removed by a simple scaling procedure. We have shown this for previously calculated widths and we have resolved an ambiguity.

A. S-C. C. thanks the Smithsonian Institution for International Exchange Scholarships and the Physical Science Research Fund of the University of Hong Kong for financial support. D. K-W. M. thanks the Croucher Foundation for his Studentship. The work of M. J. J. and A. D. was supported by the The Division of Atmospheric Sciences, The National Science Foundation. The hospitality of the Institute for Theoretical Atomic and Molecular Physics is acknowledged. The Institute is supported by a grant from the National Science Foundation to the Smithsonian Institution and Harvard University. a) Permanent address: Department of Computing Science, University of Glasgow, Glasgow G12 8QQ, UK.

${ }^{1}$ M. L. Sink and A. D. Bandrauk, J. Chem. Phys. 66, 5313 (1977); J. E. Frederick and R. D. Hudson, J. Mol. Spectrosc. 74, 247 (1979).

${ }^{2}$ H. P. F. Gies, S. T. Gibson, D. G. McCoy, A. J. Blake, and B. R. Lewis, J. Quant. Spectrosc. Radiat. Transfer 26, 469 (1981); B. R. Lewis, L. Berzins, J. H. Carver and S. T. Gibson, ibid. 36, 187 (1986); B. R. Lewis, L. Berzins, and J. H. Carver, ibid. 37, 229, 243 (1987).

${ }^{3}$ A. S-C. Cheung, K. Yoshino, D. E. Freeman, R. S. Friedman, A. Dalgarno and W. H. Parkinson, J. Mol. Spectrosc. 134, 362 (1989); D. E. Freeman, A. S-C. Cheung, K. Yoshino, and W. H. Parkinson, J. Chem. Phys. 91, 6538 (1990); A. S-C. Cheung, K. Yoshino, J. R. Esmond, S. S-L. Chiu, D. E. Freeman, and W. H. Parkinson, ibid. 92, 842 (1990); S. S-L Chiu, A. S-C Cheung, K. Yoshino, J. R. Esmond, D. E. Freeman, and W. H. Parkinson, ibid. 93, 5539 (1990).

${ }^{4}$ S. S-L. Chiu, A. S-C. Cheung, M. Finch, M. J. Jamieson, K. Yoshino, A. Dalgarno, and W. H. Parkinson, J. Chem. Phys. 97, 1787 (1992).

${ }^{5}$ P. S. Julienne and M. Krauss, J. Mol. Spectrosc. 56, 270 (1975); P. S. Julienne, ibid. 63, 60 (1976).

${ }^{6} \mathrm{H}$. Lefebvre-Brion and R. W. Field, Perturbations in the Spectra of Diatomic Molecules (Academic, New York, 1986); X. Yang, A. M. Wodtke, and L. Huwel, J. Chem. Phys. 94, 2469 (1991); P. C. Cosby, H. Park, R. A. Copeland, and T. G. Slanger, ibid. 98, 5117 (1993); A. S-C. Cheung, D. K-W. Mok, M. J. Jamieson, M. Finch, K. Yoshino, A. Dalgarno, and W. H. Parkinson, ibid. 99, 1086 (1993).

${ }^{7}$ R. S. Mulliken, J. Chem. Phys. 33, 247 (1960).

${ }^{8}$ W. H. Miller, J. Chem. Phys. 48, 464 (1968).

${ }^{9}$ M. S. Child, J. Phys. B 13, 2557 (1980).

${ }^{10}$ M. S. Child, J. Mol. Spectrosc. 45, 293 (1973).

${ }^{11}$ K. P. Huber and G. Herzberg, Molecular Spectra and Molecular Structure. IV Constants of Diatomic Molecules (Van Nostrand Reinhold, New York, 1979); A. S-C. Cheung, K. Yoshino, W. H. Parkinson, and D. E. Freeman, J. Mol. Spectrosc. 119, 1 (1986). 